\title{
The Cambodian Khmer Rouge Child Survivor and Effective Mental Health Treatments
}

\author{
Stephanie Hiromi Yamada-Mitsuuchi \\ California Southern University, Costa Mesa, CA, USA \\ Tara Rava Zolnikov ${ }^{1}$ \\ California Southern University, Costa Mesa, CA, USA \\ National University, Department of Community Health, San Diego, CA, USA
}

\begin{abstract}
During the Khmer Rouge period (1975-1979), an estimated 1.7 million people died from executions, starvation, or disease; children older than age seven were separated from their parents and suffered from a myriad of traumas, likely resulting in various adverse childhood experiences. This research was to identify the effects of trauma on the Khmer Rouge child survivor, and to assess the effectiveness of mental health treatment. A qualitative phenomenological study using 20 semistructured interviews of Khmer Rouge child survivors who immigrated to the U.S. and received mental health services. Most survivors appeared to have a secured attachment/relationship to an adult figure and had goals or an optimistic view which helped improve outcomes; most survivors experienced improved psychosocial outcomes after receiving mental health services. Participants recognized the link between mental health and physical health, while also reporting that they needed to help themselves get better in order to help their children. They reported using Dharma talk, mindfulness meditation, and mindfulness breathing to cope and manage their symptoms. These results could be transferable to other Cambodian child survivors, and other survivors of worldwide atrocities providing preventative and integrated care resulting in improved physical and mental health for this population.
\end{abstract}

KEYWORDS: Khmer Rouge, child, mental health treatments, conflict, health, treatment.

Phnom Penh, the capital of Cambodia was overthrown by the Cambodian communist group called the Khmer Rouge in 1975, resulting in a devastating attack on the Cambodian population (Hinton et al., 2011). Approximately half a million people died during the civil war from March 1970 to April 1975 (Hinton et al., 2011), and for three and half years afterwards, from April 1975 to January 1979, an additional 1.7 million people died from execution, starvation, or disease (Chan, 2015). This resulted in the elimination of more than one quarter of the Cambodian population-in fact, this atrocity is characterized as one of the most brutal and extreme episodes against humanity (Reicherter \& Ayward, 2011).

\footnotetext{
${ }^{1}$ Corresponding Author: Researcher at California Southern University, Costa Mesa, CA and National University, Department of Community Health, San Diego, CA, USA. E-Mail: tarazolnikov@ gmail.com
} 
The Khmer Rouge's goal was to erase any signs of capitalism and therefore create a "new society"; as a result, educated people, including monks and teachers, were killed, and the remaining population was sent to the rural countryside. One of the many tactics used to create the "new society" was to eliminate family loyalty and emotional attachments by separating children from their parents (Chan, 2015). If children were successfully separated from their parents, they were considered morphable and re-educated to be indoctrinated into the new society. The children were encouraged to betray their parents and tell regime leaders if their parents were not obeying the communist rules, which contributed to a lack of trust in the parent child dyad, and contributed to role reversal, wherein parents did not trust their children either. This increased the probability of negative mental health outcomes for the children (Gordon, 2016), and affected the child's ability to form a secure attachment to a caregiver and affecting their exposure to a parental role model (Field, 2011).

While adults were certainly affected by these actions, children were the most vulnerable of the Cambodian survivors. They not only experienced pain and torture associated with the Khmer Rouge, but were exposed to significant trauma in the refugee camps, such as lack of adequate food supply, poor sanitation, medical care, rape, separation from family members and even death (Chan, 2015). The only benefit occurred when children and some families were accepted as refugees and migrated to the United States; that said, the trauma continued, as they struggled in their new homeland. Adverse outcomes associated with living abroad were primarily related to their parent's inability to adjust and provide necessary basic human rights (e.g., food, shelter, etc.), which was directly associated with the parent's experiences during the Khmer Rouge (Juang et al., 2018). At this point, many children were placed in a role reversal position and took the primary caretaking responsibility for their families; this was likely because the children could adapt easier to their new environment and culture, learning the language and ultimately, becoming interpreters for their parents. In addition to these familial needs, children had to attend school and because of their poor English language skills and different culture, faced racial discrimination (Juang et al., 2018); moreover, in Cambodia, education was not available to everybody, so for some youth, this was their first-time attending school (Juang et al., 2018).

The way in which the Cambodian youth responded to their immigration experience depended on the quality of the attachment to their primary caregiver, as well as their age and developmental stage during their migration (Juang et al., 2018). Children who developed a secure attachment to their caregiver were able to adapt and use effective coping skills allowing them to be able to seek out support, develop relationships with others, and apply an internal working model that would help them remain optimistic and resilient (Juang et al., 2018).

Children who did not have parents and/or were not able to establish a secure attachment, suffered from indelible outcomes, exacerbated by the adverse childhood events (ACEs) experienced. Prolonged significant adversity during pregnancy or early childhood are known to have increased treatment resistant stress-related health problems later in life (National Scientific Council on the Developing Child, 2020). Other outcomes can include weakened physiological responses (e.g., immune system), vulnerabilities to later impairments in health (e.g., elevated blood pressure) and altered brain architecture (e.g., impaired neural circuits). Adverse events during childhood that are severe, recurring, or long-lasting can seriously affect major aspects of emotional functioning and lead to increased risk of interpersonal maladjustment and psychopathology (Shonkoff \& Garner, 2012).

Decades later, these children are now adults. The Cambodian children who survived the Khmer Rouge lived with their trauma and associated adverse developmental outcomes for over 40 years. It is likely these child survivors of the Khmer Rouge experienced long-lasting effects not only from their childhood, but across the lifespan as well. For example, Cambodian adult refugees 
have reported experiencing adverse health problems such as infectious diseases, pain and musculoskeletal issues, brain injuries from blows to the head, malnutrition from forced starvation, and high rates of stroke, diabetes, hypertension, and cardiovascular disease (Opaas et al., 2015). These trauma responses may be worse than reported, as effects often subside or change over time, with some more predominant symptoms masking others. Additionally, a focus on conflict-related trauma may not capture all sources of psychological distress, as stressors from daily life due to acculturation, which is just as relevant (e.g., as lack of sustenance, socioeconomic insecurity, etc.) (Cantor-Graae et al., 2014). Several adverse experiences during childhood and prolonged exposure to trauma inflict long-term negative mental, physical, and neurological consequences. In fact, adults with ACEs tend to be more reactive to stressful occurrences, both insignificant and significant, due to their own level of toxic stress. These responses in turn affect the physiologic or epigenetic pathways in their child (Le-Scherban et al., 2018), and since these experiences are stored in memory, an individual may be triggered long after the experience has passed (Chambers, 2017). Epigentics is the study of how chemical modifications change the gene expression of a cell without altering the DNA sequence. Environmental stressors have been known to increase cortisol levels, and if maintained at high levels, will affect gene expressions. Adult mental health disorders, such as depression, are associated with impaired parenting, dysfunctional parent-child interactions, and parenting attitudes and behaviors. Parental ACEs have also been found to disrupt the ability to form long-term attachments in adulthood, thereby affecting the ability to later form relationships (Anda et al., 2006).

In January 2005, the World Health Organization (WHO) executive board urged that support be given to "implement programs to repair the psychological damage of war, conflict, and natural disasters". It is now recognized that the inability to function mentally and physically as a result of political violence is a major public health problem (Reicherter \& Aylward, 2011). One of the ways to improve results and long-term outcome from survivors of the Khmer Rouge is through psychotherapy or other mental health services.

\section{Objectives}

In the Cambodian community, those who survived the Khmer Rouge likely still suffer from Post-Traumatic Stress Disorder (PTSD), even 30 years later (Wong et al., 2015). The experience of childhood trauma can disrupt the secure attachment between a parent and child as well interferes with the child's ability to formulate secure bonding. Treatment of complex trauma has been identified to have significant success when using a psychodynamic approach to address not only the trauma but also focusing on improving the cognitive, emotional, social, and relational capacity that were not fully developed due to the child's age in which the trauma exposure occurred (Price et al., 2019). A psychodynamic approach would be the most appropriate method to identify and process the individuals unconscious cause of emotional distress that can be traced back to their early childhood. The purpose of this study was to identify the effects of trauma on the Cambodian children who were exposed to the Khmer Rouge atrocities, and to assess effective mental health treatment interventions. The interviews were conducted as part of a doctoral dissertation process at California Southern University, Irvine, CA. The investigator has over twenty years of experience working in a non-profit community mental health setting with this population and has observed the mental and physical health impact of trauma on the Khmer child survivor. This information can be used to align culturally appropriate mental health treatment interventions to address the needs of this population as well as other cultures with similar backgrounds. 


\section{Methods}

A phenomenological study looks at both what was experienced as well as how it was experienced (Neubauer et al., 2019). This study used a descriptive phenomenological approach, which has been continuously described as a valuable research tool and strategy to understand the lived experiences of participants related to a phenomenon (Husserl, 1981; Marques \& McCall, 2005; Neubauer et al., 2019); the aim of this type of research is to identify common themes related to a phenomenon to understand the perspectives of people who have experienced it (Marques \& McCall, 2005). This method was utilized for this study to understand the experiences of the Cambodian adult, who were exposed to the trauma of the Khmer Rouge atrocities as a child and endured the migration process of arriving in the United States, and their personal perception of effective mental health treatments.

The research took place in 2019 in Long Beach, CA, where according to the 2010 Census, is one of the U.S. cities where the largest concentration of Cambodians resides, with a population of 44,522 (U.S. Census Bureau, 2010). Flyers describing the research were created and translated into Khmer. These recruitment flyers were distributed to various organizations in Cambodia Town located in Long Beach, CA, and the surrounding areas within Los Angeles County. Participants were recruited and screened to ensure that they met the criteria of the project: born between 19571975, resided in Cambodia from 1975-1979, migrated/immigrated to the U.S. after 1975 and received mental health services. Confidential interviews were held either in an office location or in the participant home lasting 1-2 hours. Prior to the recruitment and interview process, the study protocol was approved by the university's institutional review board. In addition to gathering demographic information, formulated questions focusing on the major thematic areas were asked: 1. The participant's experience as children during the Khmer Rouge, 2. The participants experiences after moving to the United States, and 3. The experiences of receiving mental health services. All participants gave full informed consent prior to commencement of the interview, which were audiotaped with written notations.

Khmer speaking participants who did not speak English or were limited in their Englishspeaking ability, were provided with a volunteer Khmer speaking translator. The ability of the participant to tell their story in their own language allowed comfort, which upheld the essence of the experience to be relayed. The volunteer translator assisted in the screening of participants to ensure that they met the criteria for the study and assisted with the interviewing, gathering, and transcribing the responses. Since the researcher did not speak the Cambodian language, according to the American Psychological Association (APA) Code of Ethics (2019), the researcher took reasonable steps to ensure that the translator did not have a dual relationship with the participant and ensured that they can perform the work competently. The volunteer Cambodian translators were employees of a local non-profit community mental health agency, who provide mental health translation on a daily basis.

Both female and males who were children between the ages of 0-18 during the Khmer Rouge period (1975-1979) were included to participate in the interviews. Debriefing with the volunteer translators occurred before and after the development of the interview questions and after analyzing participant responses. The participants' nonverbal resources, such as laughter and patting of the head, were also noted in the transcripts, and were represented by parentheses to insert a comment on something that occurred during the interview when the participant made these nonverbal gestures.

All interviews were recorded, transcribed, and analyzed. Based on the Moustakas (1994) phenomenological study framework, codes were manually generated from the interview questions. The codes allowed the researcher to identify themes and patterns, as well as supporting quotes. 
Codes were generated and entered in a codebook, with some identified examples as: "family," "symptoms," and "treatment." These were then reviewed for themes and patterns, which allowed the researcher to understand the experiences of the participant, their perceptions, and other phenomena related to child survivors of the Khmer Rouge. Themes were identified which represented information that appeared to provide support to the experiences of these child survivors and effective mental health treatments. This thematic analysis followed Moustakas modified Van Kaam method (Moustakas, 1994).

All qualitative research must provide measures to ensure validity of the data in the research. In this case, the researchers established trustworthiness through credibility, multiple participant perspectives, peer debriefing and review, reflexive journaling, and field notes. The trustworthiness of the qualitative data as part of the research process, upheld by making explicit biases and acknowledging subjective judgements (Creswell, 2007; Creswell \& Creswell, 2018). Credibility was gained through ensuring that all participants met the research criteria and that both female and males between the ages of 44 through 62 were all included to participate in the interviews. Credibility was gained through ensuring that all participants met the research criteria: born between 1957-1979, lived in Cambodia between 1975-1979, migrated/immigrated to the U.S. after 1979, currently residing in Los Angeles County, and received mental health services after arriving in the U.S. Peer debriefing and review occurred before and after developing interview questions and analyzing themes in the data to ensure language and translation appropriateness and accuracy. Reflexive journaling and field notes were documented in a diary, which was used to report on questions, related participant reactions and impressions of each interview, to reduce any bias during the researcher's documentation. That said, limitations in all research exists. Limitations of this study included the possibility of nontransferable results to other Khmer Rouge survivors in the world, researcher personal bias (e.g., Cambodian, mental health researcher), and research participant bias.

The study protocol and ethics review were approved by California Southern University's IRB Representative. All participants signed informed consent prior to the commencement of the interviews and audio recording. Codes were immediately assigned to every participant to ensure that their information was de-identified for confidentiality. This researcher documented the procedural steps taken in establishing the research protocols and utilized the Cambodian volunteers to review this researchers' data transcripts for accuracy by comparing the audio tapes.

\section{Results}

There were 20 participants ( 18 females and two males) recruited for this study that met the study's inclusion criteria (e.g., lived as a child during the Khmer Rouge, immigrated to the United States, and received some form of therapy). All participants resided within the Long Beach or immediate surrounding areas. Of these participants, one was single, 14 were married, three were separated/divorced, and two were widowers. There were 16 participants that had children, but four participants had no children. There were eight participants that were bilingual English and Khmer and 12 were monolingual Khmer speaking. When the Khmer Rouge overthrew Phnom Penh on April 17, 1975, two of the participants were between 0-five years old, eight were between six-13 years old, and ten were between 14-18 years old. The length of time from the end of the Khmer Rouge in 1979 to the participants immigration to the U.S. ranged from 0-43 years. The age of the participants arrival to the U.S. varied from 12-65 years old. Of these, seven were refugees and 13 were sponsored by family members. Upon arrival to the U.S., 11 came to the Los Angeles County area (ten to Long Beach, one to Koreatown, a neighborhood in Los Angeles), six arrived at other U.S. states, and three did not report. The participants who immigrated to the Long Beach area did 
so for a variety of reasons: three had family already living in the Long Beach area, two had friends living in Long Beach, and one came for medical reasons. All but one participant received traditional mental health services, or in combination with non-traditional services. There were 19 participants who received individual therapy sessions, with 11 that reported receiving psychotropic medication. The length of services ranged from three months to five years.

\section{Khmer Rouge Experience}

The experiences of participants from the Khmer Rouge period to present were very similar. Except for the two participants who were between 0 -five years old during the Khmer Rouge period, all memories focused on facing starvation, exposure to death and executions, long days of hard labor, and struggles of living in fear. "I was [supposed] to be killed because I stole food to feed my younger sibling who was only 1 years old" (PT08). The younger participants also discussed their hardships and how they experienced family struggles. "Mom had a [gold] ring and [sold it] to buy us rice and a cow... No one gave us food or work; we had to provide for ourselves" (PT18). Despite the trauma at that time, some were able to express optimism about their future in the U.S. "If I could survive the killing fields genocide, I can make it here" (PT12). This encouraged resilience and the ability to make life the best it could be, albeit childhood trauma experiences.

\section{Life in the United States}

Life for the participants was primarily described as difficult, mostly due to language and cultural barriers. Even those who knew some English when they arrived, voiced difficulties with the language and adjusting to western culture.

The White people would tease us badly. They threw food at us; they were mean. I told them I was from Cambodia and that it was very far away. They told me my eyes were small. The food was different, and I didn't know how to eat it. They would push food to me and I would push it back. [They once threw] spaghetti on my head and the school called my sponsor to pick me up. It got really bad at school, so my sponsor put me with my brother and sister at the same school. We were used to eating rice, but there were no Asian stores [nearby to buy rice]. My sponsor would cook food, but we didn't eat it because we didn't know what it was. (PT13)

Loneliness, hopelessness, excessive worries, isolation, and loss of independence were common themes with the participants which contributed to their mental health symptoms; most indicated that these symptoms began when they arrived in the U.S. "I have [had] so many kinds of symptoms, such as anxious thoughts, panic attacks, unable to control and function, irritable, [tightness in my] head, and [had] a hard time [removing] worries" (PT10).

Goals and optimism helped improve perspectives and created actions towards making life better. These participants made overall improvements to their mental health and living situations in the U.S. "I had inner strength to continue. I found [out] that all bad things [can] not continue. Keep going and do your best. When good deeds happen[ed], I [felt] blessed for the future" (PT11). In fact, participants who no longer receive mental health services, are financially secure through employment, continue to utilize coping skills that they learned as children. 
I'm Buddhist and depend on destiny and [how] what has happened in the past affects current life. When good deeds happen, I feel blessed for the future. I need to remember to check my feelings daily and to [live] in the moment. (PT11)

\section{Mental Health and Treatment}

All participants received mental health services but did not seek services on their own. Participants reported that they were referred to mental health services by somebody that they knew and trusted. "I decided to enroll in mental health services because I want to feel relief from my depression. I know about mental health services via CAA [Cambodian Association of America]" (PT10). Another participant suggested another source of mental health information. "I knew of mental health services via TCC [The Children's Clinic]. I was interested in services because of my stress and crying, problem sleeping, and anxious thoughts" (PT8).

There were three themes and outcomes that arose, as participants described resulting symptoms as anxiety, depression, and PTSD. People who witnessed executions and killings of family members described the most severe mental health symptoms, but all reported using similar coping mechanisms. These include: Dharma talk, meditation, mindfulness breathing, going to temple, participating in activities where others share their same language and culture, and understanding the importance of remembering to take psychotropic medication.

I am trying to function better by blocking my thoughts and focusing on Dharma talk, which [focuses on] life. I am trying to understand [more] about life. I realized that I cannot [exit the past] if I keep thinking a lot. (PT10)

Those who experienced poor memory claimed that although they could not recall what they learned in their sessions with the therapist, they remembered how the therapist and mental health team made them feel; this led to engagement in daily activities where they were able to re-live the same feelings that they felt with the mental health treatment team, which were typically positive.

I forget a lot, but I remember to keep busy and get fresh air at the beach. I plant vegetables. When I do this, I don't think about the issues. This helps me especially when the vegetables grow and become green. It makes me happy. (PT05)

Participants also acknowledged their improved symptoms due to the flexibility of the therapist, the use of cultural expressions during treatment sessions, the support of a mental health team, and home visitations.

The counselor comes to visit me, and I have a chance to [relieve] my pain. My counselor has taught me a lot, but I cannot remember [everything]. I have a lot of forgetfulness. However, I feel more connected and support from the services. (PT20) 
In addition, participants indicated that their involvement in social activities where they interacted with others who speak their language and who also receive mental health services contributed to their decreased sense of isolation, loneliness, and depression.

\begin{abstract}
My life is full of suffering and sadness. I experience nervousness and am worried all the time. About one year ago, I enrolled in mental health services, and I feel very supported. I meet my counselor once a week. I am able to [stop feeling] pain by hang[ing out] with others, participating in the activities such as cooking class or support group, [and] having emotional support from church. (PT20)
\end{abstract}

Participants believed that their mental health affected their physical health, and that this influenced their children. "My daughter used to have asthma and now, she does not need to use inhaler [anymore]. I think [one reason for this is] because I have learned to manage my symptoms [and that has positively affected her]" (PT11). Participants indicated that they need to care for themselves before they could care for others, which is a shift in the historical thinking of family honor, whereby they tended to care for others before themselves. "If we don't have emotional health, [our loved ones] will take after us, so we need to help ourselves first" (PT15). They would enroll their children in mental health services, so they do not suffer the same as them. "I think that the way to help my kids is to enroll them for mental health service like me" (PT16).

\title{
Discussion
}

The Khmer Rouge was one of the worst crimes against humanity, wherein the Cambodian population encountered outlandish views which were paired with psychological and inhumane suffering. Participants in this study reported personal accounts of starvation and exposure to death during the Khmer Rouge; upon arrival to the U.S., these participants reported poor living conditions, lack of independence, cultural and linguistic barriers, and isolation. Since the Khmer Rouge, the Cambodian refugee/immigrant arriving in the U.S. continued to show signs of PTSD and depression due to traumatic experiences not only from the atrocities they endured during this period, but also with the social and cultural transition faced by moving to a new country. According to Marshall and colleagues (2005), an estimated 62\% of Cambodians refugees in the U.S. suffered from PTSD, and 51\% suffered from depression. The results of this qualitative study confirmed these rates, as most of the participants self-described having symptoms of PTSD, depression, and anxiety.

Interestingly, Marshall's (2005) also conducted research on the Cambodian population in Long Beach 24-26 years after the 1979 fall of the Khmer Rouge and found that this population continued to suffer from chronic mental and physical adverse health effects. Wong et al. (2015) study did not address effective mental health treatments used by participants to help manage their reported symptoms. However, in this study, participant's symptoms were described as severe, including flashbacks, prolonged distress, sleep disturbance, inability to focus or remember. Their reports of improved mental health or physical health primarily centered around how they felt and appeared to focus around their ability to be connected and supported. In this study, all participants reported having a chronic illness, and half of the participants were able to identify how their mental health contributed to their physical health. The participants were able to explain the importance of using their coping skills in order to reduce the severity of mental symptoms and they reported that their physical health had also improved. This study also found that participants who reported 
experiencing PTSD symptoms were directly exposed to traumatic events such as witnessing executions and watching others die from starvation, shootings, mine explosions, or illness.

Various treatment modalities such as Acceptance Commitment Therapy (ACT) and Narrative Exposure Therapy, could be possible effective treatments for the Khmer Rouge survivor. ACT is a therapeutic intervention based on Relational Frame theory which uses mindfulness along with other aspects of Buddhism and Narrative Exposure Therapy is a short-term trauma treatment approach that integrates Cognitive Behavioral Therapy and Testimony Therapy. ACT was studied on a small population of Cambodians and hold aspects that are congruent with their cultural beliefs, such as mindfulness and focusing on body sensations, or somatic symptoms (Burckhardt et al., 2017). Almost all the participants in this study reported that they used similar practices found in ACT, such as Dharma talk, mindfulness breathing, gardening, and staying in the present to help them manage their symptoms, and thus improving how they feel both physically and mentally. They also reported the need to remember to use their coping skills in order to keep their symptoms from returning.

Early intervention to prevent or reduce the likelihood of intergenerational trauma, would likely have a positive impact on the well-being of this population. In this research, participants reported using coping skills such as being in the present, letting go of what one has no control over, and mindfulness techniques. This is supportive of Buddhist teaching, and supports the techniques used in the ACT model. Thus, incorporating the spiritual beliefs of this population into their mental health treatment was beneficial. Whether or not the participants were Buddhist, they reported using these techniques.

The integration of treatment models may provide better results for treating the Cambodian Khmer Rouge child survivor. In Cambodia, the Transcultural Psychosocial Organization (TPO) combines Buddhist ceremonies, whereby the survivors voice their losses to the monk and this sanctity is incorporated as part of their trauma counseling and self-help groups (Ebrahim, 2015). Although Buddhism is the primary religion in Cambodia, not all the participants reported being Buddhist. However, most of the participants reported using Dharma-talk as their way to cope to maintain better mental health. The participants explained that Dharma-talk focused on how to "stay focused", "let it go", "don't worry about what you can't control", which are teachings from Buddhist practice. The blending of Buddhism and narrative therapy was shown to be more effective at reducing PTSD symptoms (Strasser et al., 2014). In addition to Dharma-talk, participants reported being involved in temple activities, not only for praying, but to socialize, and they reported feeling better with improved mental health symptoms. The participants also reported that journaling and thinking positive thoughts was a way of coping. These coping mechanisms are supportive of the TPO's use of Buddhism and narrative therapy.

The use of complementary and alternative medicine (CAM), or traditional complementary and alternative medicine (TCAM) were often used by the participants in this study. Complementary and alternative medicine (CAM) is health care practices or interventions that are in addition to or an alternative to standard treatment. Traditional complementary and alternative medicine (TCAM) are health care practices that are indigenous to different cultures and is used interchangeably with CAM. Prayer, meditation, relaxation, yoga, and tai chi were used as coping mechanisms in addition to their mental health treatments, which is supportive of the findings from Peltzer et al. (2016), Yi et al. (2017), and Berthold et al. (2007). The participants reportedly continued to use these techniques as daily coping mechanisms outside of treatment, and after their mental health treatment had ended. There were two participants who reported using only CAM, however, one reported that her symptoms did not improve as she indicated that she was still hearing voices; this participant sought mental health services while continuing to use CAM and reported improved symptoms when she utilized both (PT10). 
Dharma-talk was reported as helping the participants reduce their symptoms and can be compared to attachment theory. The Eastern philosophy of Buddhism and the ability of letting go, releasing things that are unhealthy or that causes distress is believed to be similar to having a secure attachment; individuals with secure attachments are believed to be able to perceive relationships more objectively and are able to let go when situations cause them distress. The bond between infant and caregiver in forming a stable and trusting relationship will lay the foundation for adult attachment with implications for social and emotional outcomes later in life (Chambers, 2017). Through their stories, the participants attachment to a parent figure was evident, as well as their future optimistic outlook of hope. In general, these participants performed better than others in this study after experiencing their traumatic events. These adults suffered mental health and physical health symptoms, but the focus of their stories was not of pain and suffering, but of optimism that they are almost at their goal of having a better life. This supports the findings by Maschi et al. (2012), indicating that positive attitude or optimism, and social supports contribute to overcoming childhood trauma; social supports help buffer stress.

Some barriers and suggested improvements were also mentioned. Several participants who received mental health services from other agencies, reported discontinuing services due to lack of flexibility by the therapist, inability to get to the agency, and forgetting appointments due to memory problems. These impediments to services could be remedied by using community health workers to help the individual access needed mental health services. The participants reported that they felt understood and supported by their therapists even if translators were used. They also reported receiving reminder calls for appointments, reinforcement of what they learned, help with monitoring their medication, and positive encouragements which contributed to their overall wellness. Due to the participants reports of feelings of loneliness and isolation, and feelings of lack of support from their family, this model contributed to the overall improvement of their symptoms, and supportive of findings by Shannon and colleagues (2016).

\section{Implications for Professional Practice}

By collecting and analyzing the data of these participants, this study provided an understanding of the experiences of the Cambodian individual who experienced the Khmer Rouge as children, how they managed their mental health symptoms, and what behavioral health treatments were effective in helping to improve their symptoms. Most participants were able to recall their childhood memories of what occurred during the Khmer Rouge period (1975-1979), such as memories of starvation, hard labor, and exposure to death and dying. Those who were too young, were able to recall their childhood memories of their life in Cambodia with their parents who experienced the Khmer Rouge, describing how their parents worked hard and used harsh discipline. All were able to express their experience of migration to the U.S. and their difficulties adjusting to the cultural differences.

Participants were able to share what they deemed as effective mental health care, and how they continue to maintain an improved sense of mental health, which improves their physical health. Although most participants continue to receive mental health treatment, they indicated improved symptoms since treatment began, and consistently indicated improved mental health due to:

- The use of "dharma talk" and mindfulness in learning to be "present" helped in regulating and managing symptoms 
- The blending of traditional and non-traditional treatments was culturally relevant and easier for the participant to understand and relate

- The flexibility in scheduling treatment sessions helped to reduce participants anxiety of not remembering their appointments

- Home visitations reduced not only barriers to accessing services but reduced their sense of isolation and loneliness which helped improve their symptoms.

This information suggests that interventions which focus on improving emotional regulation skills may provide an efficient treatment strategy for both psychological and physical health problems. Emotional regulation can be defined as the individual's ability to recognize, monitor, express, and modify emotional reactions in a manner that facilitates adaptive functioning (Cloitre et al., 2018).

Trauma-informed care among those who have potential contact with this population, such as health professionals, educators, faith-based, child welfare and welfare benefits offices would especially benefit this population as their symptoms are somaticized and may respond with a trauma reaction, such as complaining of headaches, stomachaches, or panic attacks. The Cambodian Symptom and Syndrome Inventory (C-SSI) identified Cambodian trauma responses and linked them to somatic complaints, including dizziness, headaches, neck soreness, or shortness of breath, which have been translated into cultural syndromes of "khyal attacks", "thinking too much", or having a "ghost pushing you down" (Hinton et al., 2013, p. 348). These types of complaints can essentially be translated by people who are more aware of the cultural setting in which they originate. This confirms the importance of having outside sources help and assist.

Moreover, participants in this study did not seek mental health services on their own but accessed mental health services as a result from meeting someone who knew about mental health, sequentially sought care, and later reported improved mental health and physical health symptoms. The participants in this study were identified by non-mental health workers who encountered the participant through their work settings, whether it was through assisting the participant with an application, disseminating general information on services in the community, or a visit with their primary care physician. In many cases, the community worker who helped the participant to access other services, such as government or medical benefits, was viewed as a trusted individual. The ability to recognize signs of trauma and to provide psychoeducation on how trauma affects the mental and physical health, can help engage an individual to seek needed services. Also, having a list of accessible mental health resources and following up to make sure that services were appropriately accessed would help to connect the individual to helpful programs.

Implementation of home visitation and a team approach to service delivery would be beneficial for populations who are isolated, not only by geographic location, but due to language and culture. As supported by the findings by Berthold et al. (2018), community health workers who are bilingual in Khmer and English, are not only able to check on isolated individuals, but are able to advocate and help bridge the cultural gap for them. These community workers develop a relationship with the isolated individual because they visit on a regular basis. The participants in this study reported that they received services by a team of mental health workers, which consisted of a therapist, a case manager, and a peer advocate, all of whom visited them at home through a coordinated care approach.

The use of blended treatments of non-traditional and traditional services would benefit individuals who hold traditional values and beliefs. The use of culture specific examples and/or symbols have more meaning to patients and are easier for the patient to comprehend, rather than using western terminologies and examples (Hinton \& Patel, 2018). The results of this study confirm 
the connection that participants felt to their therapist because they understood their culture, using culture specific examples and explanations. For example, one participant specifically mentioned how they first requested help from a monk, but after continued suffering from symptoms related to trauma, sought traditional mental health services and temple services with much success.

\section{Limitations}

This study only included research on Cambodian participants who were between the age of 0-18 during the Khmer Rouge period (1975-1979), who experienced trauma as a result of the Khmer Rouge, and subsequent trauma as refugees/immigrants to the U.S. The study did not capture experiences of those over the age of 18 during the Khmer Rouge period, and only those who received mental health services either traditionally or non-traditionally were included. Last, participants were limited to those residing in Long Beach, California, and the surrounding areas.

Possible limitations to the study included: retrospective data whereby the participants needed to recall their experience from 40 years prior, third parties, social desirability on the part of the participant, inefficiency in the interpretation, English as a second language, or deficiency in the translation of the responses from the participant, interpreter, and transcriptionist. The issue of third parties was addressed by declining outside influence or direction, aside from the three Cambodian volunteers used for this study. Social desirability could be considered a limitation, as participants could answer the questions in a way to please the interviewer, Khmer interpreter, or other family members who were present during the interview. The interpreter and the audio-tape translator reviewed and re-listened to the audio tapes and provided an accurate translation for the transcription by the researcher. The use of a Khmer interpreter to English was used because of the limited command of the English language of the participant and allowed the participant to tell their story in their primary language so as not to lose the "flavor" of their experience.

\section{Conclusions}

Severe adverse experiences and prolonged exposure to trauma during childhood inflict long-term negative mental, physical, and neurological consequences as seen with the participants in this study. The results of this study have significant implications for the Cambodian community, who suffered from one of the world's worst crimes against humanity. The atrocities experienced by the Cambodian child survivor of the Khmer Rouge for the past 40 years have carried a great burden on the psyche and the health of its people. This study focused on the child survivor of the Khmer Rouge who immigrated to the U.S., and who received mental health services. Many studies have been conducted on refugees and trauma, some focusing on treatment modalities. But very few studies have been done specifically on this study's population with their personal accounts on what they perceived as helping them improve their mental health symptoms.

The outcome of this study highlights the importance of community, traditional values, and support needed to relieve chronic suffering from the trauma. This type of information can provide solutions to improve the quality of life for Cambodians affected by the Rouge, though these suggestions may be transferable and provide insight to encourage positive change for children exposed to war. Although this research paper focused on the Cambodian child survivors of the Khmer Rouge, children in other war-torn countries are reported to have high prevalence of PTSD symptoms. Syrian, Palestinian, and Bosnian refugee children were reported to have similar rates of PTSD (45\%) (Sirin \& Rogers-Sirin, 2015) and psychosomatic reactions were found in Nazi Holocaust child survivors similar to the Cambodian child survivor (Uy, 2018). In 2005, the WHO urged support to implement programs that will repair the psychological damage of war, conflict, 
and natural disasters. With the increasing numbers of children being exposed to war and violence, effective interventions to improve their mental well-being should be our focus in order to thwart the future public health problem (Reicherter \& Aylward, 2011).

I never expected that we are able to live here in America. Ifeel very happy, have a big dream if we are able to have all my kids to start life over here as well; life is full of justice and [is] fair. (PT03)

\section{Funding Details}

No funding was received for this research.

\section{Disclosure Statement}

The authors confirm no financial interests or benefits from the application of this research.

\section{References}

American Psychological Association (2019). Ethical principles of psychologists and code of conduct. https://www.apa.org/ethics/code

Anda, R. F., Felitti, V. J., Bremner, D., Walker, J. D., Whitfield, C., Perry, B. D., Dube, S. R., \& Giles, W. H. (2006). The enduring effects of abuse and related adverse experiences in childhood: A convergence of evidence from neurobiology and epidemiology. European Archives Psychiatry and Clinical Neuroscience, 256, 174-186. https://doi.org/10.1007/s00406-005-0624-4

Berthold, S. M., Kong, S., Ostrander, J., \& Fukuda, S. (2018). Socially isolated Cambodians in the U.S.: Recommendations for Health Promotion. Advances in Social Work, 18(3), 808-832. https://doi.org/10.18060/22318

Berthold, S. M., Wong, E. C., Schell, T. L., Marshall, G. N., Elliott, M. N., Takeuchi, D., \& Hambarsoomians, K. (2007). U.S. Cambodian refugees' use of complementary and alternative medicine for mental health providers. Psychiatric Services, 58(9), 1212-1218. https://www.researchgate.net/profile/S_Berthold/publication/6073089_US_Cambodian_R efugees\%27_Use_of_Complementary_and_Alternative_Medicine_for_Mental_Health_Pr oblems/links/561d617b08aef097132b2282/US-Cambodian-Refugees-Use-of-

Complementary-and-Alternative-Medicine-for-Mental-Health-Problems.pdf

Burckhardt, R., Manicavasagar, V., Batterham, P. J., Hadzi-Pavlovic, D., \& Shand, F. (2017). Acceptance and commitment therapy universal prevention program for adolescents: a feasibility study. Child and Adolescent Psychiatry Mental Health, 11(27). https://doi.org/10.1186/s13034-017-0164-5

Cantor-Graae, E., Chak, T., Sunbaunat, K., Jarl, J., \& Larsson, C. A. (2014). Long-term psychiatric consequences of exposure to trauma in Cambodia: A regional household survey. Social Science and Medicine, 123, 133-140. https://doi.org/10.1016/j.socscimed.2014.10.049

Chambers, J. (2017). The neurobiology of attachment: from infancy to clinical outcomes. Psychodynamic Psychiatry, 4(4), 542-563.

Chan, S. (2015). Cambodians in the United States: Refugees, immigrants, American ethnic minority. In Oxford research encyclopedia of American history. https://doi.org/10.1093/acrefore/9780199329175.013.317

Cloitre, M., Khan, C., Mackintosh, M., Garvert, D. W., Henn-Haase, C. M., Falvey, E. C., \& Saito, 
J. (2018). Emotion regulation mediates the relationship between ACES and physical and mental health. Psychological Trauma: Theory, Research, Practice, and Policy. https://doi.org/10.1037/tra0000374

Creswell, J. (2007). Qualitative inquiry and research design: Choosing among five traditions (2nd ed.). SAGE Publications.

Creswell, J. W., \& Creswell, J. D. (2018). Research design: Qualitative, quantitative, and mixed methods approaches (5th ed.). SAGE Publications.

Ebrahim, S. (2015). Political psychology, identity politics, and social reconciliation in postgenocidal Cambodia. Global Societies Journal, 3(0), 60-68. https://escholarship.org/uc/item/8s1938qf

Field, N. P. (2011). Intergenerational transmission of trauma stemming from the Khmer Rouge regime: An attachment perspective. In B. Van Schaack, D. Reicherter, \& Y. Chhang (Eds.), Cambodia's hidden scars: Trauma psychology in the wake of the Khmer Rouge (pp. 70-91). Documentation Center of

Cambodia. https://papers.ssrn.com/sol3/papers.cfm?abstract_id=2758130

Gordon, N. (2016). NGOs, international aid, and mental health in Cambodia [Master's thesis, University of Washington]. http://hdl.handle.net/1773/35512

Hinton, D. E., \& Patel A. (2018). Culturally sensitive CBT for refugees: Key dimensions. In N. Morina \& A. Nickerson (Eds.), Mental health of refugee and conflict-affected population (pp. 201-219 ). Springer. https://doi.org/10.1007/978-3-319-97046-2_10

Hinton, D. E., Kredlow, A., Pich, V., Bui, E., \& Hofmann, S.G. (2013). The relationship of PTSD to key somatic complaints and cultural syndromes among Cambodian refugees attending a psychiatric clinic: The Cambodian somatic symptom and syndrome inventory (CSSI). Transcultural Psychiatry, 50(3), 347-370. https://doi.org/10.1177/1363461513481187

Hinton, D., Hinton, A., Eng, K., \& Choung, S. (2011). PTSD severity and key idioms of distress among rural Cambodians: The results of a needs assessment survey. In B. Van Schaak, D. Reicherter, \& Y. Chhang (Eds.), Cambodia's hidden scars: Trauma psychology in the wake of the Khmer Rouge (pp. 47-68). Documentation Center of Cambodia.

Husserl, Edmund G. (1981). Pure phenomenology, its method, and its field of investigation. In Peter McCormick \& Frederick A. Elliston (eds.),_Husserl: Shorter Works_. University of Notre Dame Press.

Juang, L. P., Simpson, J. A., Lee, R. M., Titzmann, P. F., \& Schachner, M. K. (2018). Using attachment and relational perspectives to understand adaptation and resilience among immigrant and refugee youth. American Psychologist, 73(6), 797-811. https://doi.org/10.1037/amp9999286

Le-Scherban, F., Wang, X., Boyle-Steed, K. H., \& Pachter, L. M. (2018). Intergenerational associations of parent adverse childhood experiences and child health outcomes. Pediatrics, 141(6),. https://doi.org/10.1542/peds.2017-4274

Marques, J.F. \& McCall, C. (2005). The application of interrater reliability as a solidification instrument in a phenomenological study. The Qualitative Report, 10(3) 439-462. http://www.nova.edu/ssss/QR/QR10-3/marques.pdf

Marshall, G. N., Schell, T. L., Elliott, M. N., Berthold, M., \& Chun, C. (2005). Mental health of Cambodian refugees 2 decades after resettlement in the United States. Journal of American Medical Association, 294(5), 571-579. https://doi.org/10.1001/jama.294.5.571

Maschi, T., Baer, J., Morrissey, M. B., \& Moreno, C. (2012). The aftermath of childhood trauma on late life mental and physical health: A review of the literature. Traumatology, 1-16. doi: $10.1177 / \mathrm{I} 532765612437377$

Moustakas, C. (1994). Phenomenological research methods. SAGE Publications. 
National Scientific Council on the Developing Child (2020). Connecting the brain to the rest of the body: Early childhood development and lifelong health are deeply intertwined. (Working Paper no. \#15). www.developingchild.harvard.edu

Neubauer, B.E., Witkop, C.T., \& Varpio, L. (2019). How phenomenology can help us learn from the experiences of others. Perspect Med Educ 8(2), 90-97. https://doi.org/10.1007/s40037019-0509-2

Opaas, M., Psychol, C., Varin, S., \& Philos (2015). Relationships of childhood adverse experiences with mental health and quality of life at treatment start for adult refugees traumatized by pre-flight experiences of war and human rights violations. The Journal of Nervous and Mental Disease, 203(9), 684-695. https://doi.org/10.1097/NMD.0000000000000330

Peltzer, K., Pengpid, S., Puckpinyo, A., Yi, S., \& Anh, L. V. (2016). The utilization of traditional, complementary, and alternative medicine for non-communicable diseases and mental disorders in health care patients in Cambodia, Thailand, and Vietnam. BioMed Central Complementary and Alternative Medicine, 16(92), 1-11. https://doi.org/10.1186/s12906016-1078-0

Price, M., Arditi, R., Olezeski, C., \& McMahon, T.J. (2019). Psychological assessment and treatment of emerging adults exposed to complex trauma. Evidence-Based Practice in Child and Adolescent Mental Health, 4(1), 273-295. https://doi.org/10.1080/23794925.2019.1618225

Reicherter, D., \& Aylward, A. (2011). The impact of war and genocide on psychiatry and social psychology. In B. Van Schaack, D. Reicherter, \& Y. Chhang (Eds.), Cambodia's hidden scars: Trauma psychology in the wake of the Khmer Rouge (pp. 14-32). Documentation Center of Cambodia.

Shannon, P.J., Vinson, G.A., Cook, T., \& Lennon, E. (2016). Characteristics of successful and unsuccessful mental health referrals of refugees. Administration and Policy in Mental Health, 43(4), 555-568. https://doi.org/10:1007/s10488-015-0639-8

Shonkoff, J. P., \& Garner, A. S. (2012. The lifelong effects of early childhood adversity and toxic stress. American Academy of Pediatrics, 129(1), 232-246. https://doi.org/10.1542/peds.2011-2663

Sirin, S. R., \& Rogers-Sirin, L. (2015). The educational and mental health needs of Syrian refugee children. Migration Policy Institute. https://www.researchgate.net/publication/287998909_The_Educational_and_Mental_Hea lth_Needs_of_Syrian_Refugee_Children

Strasser, J., Chhim, S., \& Taing, S. (2014). Narrative exposure therapy (NET): Culturally sensitive trauma treatment for Khmer Rouge survivors. The Journal of the International Association of Buddhist Universities, $\quad 7(1), \quad 1-5$. http://www.ojs.mcu.ac.th/index.php/jiabu/article/view/781/660

U.S. Census Bureau (2010). American Fact Finder. https://factfinder.census.gov

Uy, K. K., \& Okubo, Y. (2018). Reassembling a shattered life: A study of posttraumatic growth in displaced Cambodian community leaders. Asian American Journal of Psychology, 9(1), 4761. https://doi.org/10.1037/aap0000111

Wong, E. W., Marshall, G. N., Schell, T. L., Berthold, M., \& Hambarsoomians, K. (2015). Characterizing the mental health care of U.S. Cambodian refugees. Psychiatric Services, 66(9), 980-984. https://doi.org/10.1176/api.ps.201400368

World Health Organization (2001). The World health report: 2001: Mental health: New understanding, new hope. U.S. Government Printing Office.

Yi, S., Ngin, C., Tout, S., Chhoun, P., Fleming, T., \& Brody, C. (2017). Utilization of traditional, complementary and alternative medicine and mental health among patients with chronic 
diseases in primary health care settings in Cambodia. International Journal of Mental Health Systems, 11(58), 1-13. https://doi.org/10.1186/s13033-017-0167-x

\section{Notes on Contributors}

Stephanie Yamada-Mitsuuchi is a post-doctoral intern working with infants and young children who have been exposed to trauma. She has spent the majority of her career providing services in a community mental health setting and has tremendous interest in the area of early childhood trauma and effective treatments. E-Mail: smitsuuchi@earthlink.net

Dr. Tara Rava Zolnikov concentrates on providing vulnerable populations with a voice and uses qualitative methods such as autoethnographic, ethnographic, and phenomenological perspectives to understand or live through these experiences to recreate them for a widespread audience. She focuses on diverse and cultural populations, who suffer indiscriminately. Dr. Zolnikov has published extensively in some of the world's most renowned public health and global health journals, including the Lancet Oncology, American Journal of Public Health, AIDS and Behavior, and Science of the Total Environment. She has also written and published two books, which focus on qualitative research and global health. E-Mail: tarazolnikov @ gmail.com ; ORCID ID\# https://orcid.org/0000-0002-0106-1214

Manuscript received October 5, 2021

Final revision received January 26, 2022

Accepted January 30, 2022 